\title{
Study on a New Type Destruction Method for Abandoned Combustion Tear Gas of CAPF
}

\author{
Dayong Jiang \\ Research Department, Engineering University of CAPF, Xi'an, 710086, P.R.China
}

Key words: Combustion tear gas; destruction method; water jet technology.

\begin{abstract}
Based on the characters of weapons and equipment, techniques and methods of destruction for anti-riot ammunition equipped by CAPF were discussed in this paper. Combing the main structure and function of common anti-riot ammunition, a new Destruction Method with water jet cutting technology was put into use for abandoned combustion tear gas through theoretical analysis and experimental verification. The study support important significance to maintenance equipment safety and improve combat effectiveness.
\end{abstract}

\section{Introduction}

Anti-riot ammunition played an important role in modern counter-terrorism operations when troops and police personnel perform tasks as the essential weapons [1,2]. With the development of sound, light, electricity, gas and other munitions technology, the types of anti-riot ammunition were also perfected, and their production, use and storage had a continuous expansion trend, but the attendant abolition of ammunition has become more and more prominent. Because the structure and characteristics of riot ammunition were widely different from the conventional ammunition, it determined that the technological methods of disposal processing were also different. The primarily aim of disposal work was to safe handle three types of abandoned anti-riot ammunition timely through the relevant technical measures, including some expired because of exceeding the storage period, existing security risks and updating equipment. Eliminating such potential safety hazard not only ensure staff and workplace safety but also encourage the smooth progress of scientific research equipment.

\section{The characteristics of disposal work for obsolete ammunition}

\subsection{Waste object}

Anti riot ammunition is a kind of non-lethal weapons, which usually achieve to operational effectiveness by releasing sound, light, smoke, fog, aerosols and other irritants by fire or explosion, not by striking the damage target generally. For this reason, the killing capacity to staff and object was limited, and the riot ammunition damage effect is less than conventional ammunition by an accident. For example, there was only a small amount black powder inside of the explosive tear gas, which limited the detonation capacity and result in the poor sympathatic detonation; combustion tear gas usually loads a small amount of propellant inside, whose main ingredient is potassium chlorate, combustion agent and a small amount of metal particles. The mode of action was often combustion, and it is difficult to reach the condition of explosion. In this paper, the most widely used combustion tear gas named Rs97-2 was studied with typical feature [3], and its structure shown in Fig.1.

The action principle was shown as following steps: When Rs97-2 tear gas was thrown by hand or gun shooting, the propellant inside can cause the irritants CS (o-chloro-benzylidene malononitrile) sublimation by burning, and the aerosols in the air formed and began to chase the crowd. Muzzle velocity is relatively small because of the indirect contact with the target, so the projectile material usually adopts aluminum that can satisfy for the strength requirement. Internal charge press-fit using two grain filling, because it contains more functional agent, the shelf life is much smaller than conventional propellant, such that the overall life expectancy is shortage of ammunition. For the 
sake of better sealing, components used thread structure among them. After assembled and bonded directly together, thread can not be molded by hand once the demolition. Metal projectile and ignition devices typically have recycling value, and should try to taken protective measures when handling in order to re-use it.

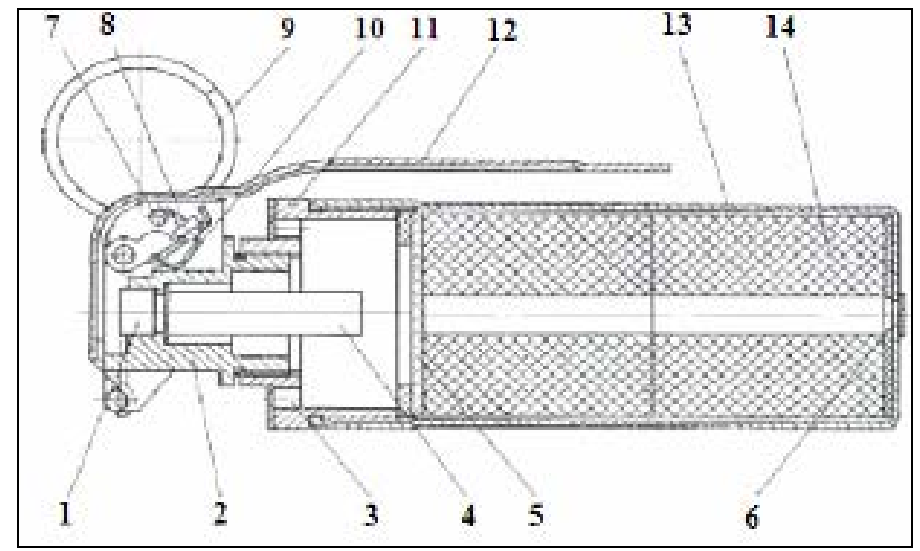

1-primer cup; 2-percussion mechanism; 3-smoke hole; 4-delayer; 5-starting mix; 6smoke hole; 7-spur guard; 8-firing pin; 9-tab; 10-striker spring;

11-connection receptacle; 12-hand grip; 13-projectile body; 14-charge

Fig.1: Construction of Rs97-2 combustion tear gas

\subsection{Requirements}

As an important part of operational equipment support, disposal work for obsolete anti-riot ammunition was also put forward higher requirements with the continuous improvement of the overall force protection capabilities. In general, technologies of disposal work for waste anti-riot ammunition should reference the latest research results for the abolition of conventional ammunition, mainly reflected in the following aspects:

(1) Study on new ammunition destruction modes including water jet cutting, incinerator and biological composting, wherein the abrasive water jet technology was generally acknowledged to have good prospects in terms of anti-riot ammunition disposal.

(2) Study on contamination control in the ammunition disposal process, such as prohibiting or restricting the large equivalent burning or blasting [4], and develop the biodegradation, adsorption, membrane treatment methods for the waste water and smoke of destruction processing.

(3) Study on automating and intelligent of disposal work for waste anti-riot ammunition, it not only effectively guaranteed the safety of the operator, but also help improve the technological level and destruction efficiency.

Based on the structural features of anti-riot ammunition, the process security should be ensured at three areas including the equipment, technology and operation. Although sensitivity of the main charge is less than high explosive, severe impact and friction is prohibited during operation. In order to prevent accidents, make sure that there is no explosive hazard nearby and as the case taken down the sensitivity by a sense of other technical measures.

\section{Introduction of destruction methods}

\subsection{Selection}

Ensuring the process safety is the premise and foundation of disposal work for anti-riot ammunition. First, classify management should be ascertained in accordance with composition, ignition, amount and types of the ammunition in suspense. High-risk or unknown-risk ammunition should be treated 
separately, and the same batch of ammunition should be processed simultaneously. Second, whether anti-riot ammunition could be demounted or not should be in accordance with the structural characteristics before choosing the most timely destruction method, shown in Figure 2.

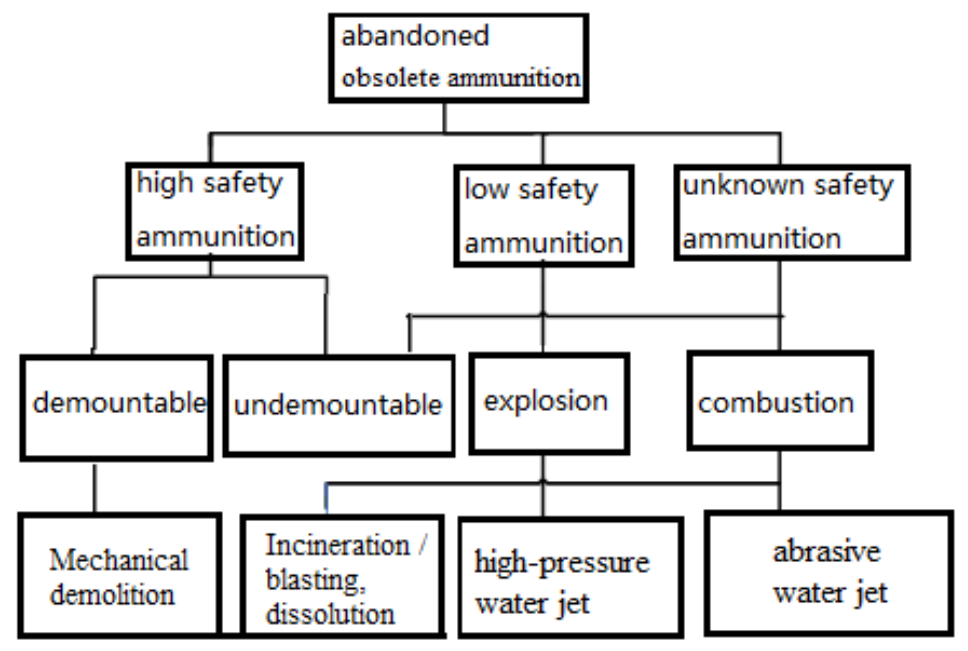

Fig.2: Classification of disposal work for obsolete ammunition

\subsection{Water jet cutting technology}

Limited by the complex internal structure, cutting efficiency is higher than the dismantling under the premise of process safety. Judged from the strength and material of projectile body, anti-riot ammunition was easier to be cut than common ammunition. Water jet cutting technology has developed in the 1970s[5,6] for the waste of ammunition emerging, because it has virtue of smokeless, odorless, non-toxic, non-sparking and generating less heat, etc. Because the water jet can inhibit the generation of local hot spots, the temperature rise of cut pieces upon impacting is small that can ensure the safety of the cutting process, especially in the case of explosive hazardous areas or operations. Therefore, at the beginning of the last twenty years, it gradually applied to deal with demilitarization of abandoned ammunition. According to the different parameters, the cutting jet technology can be divided into high-pressure water jet and abrasive water jet, whose technical parameters were in Table1.

Table1: Comparison of parameters between two water jet technologies

\begin{tabular}{|c|c|c|c|}
\hline $\begin{array}{c}\text { Serial } \\
\text { number }\end{array}$ & $\begin{array}{l}\text { technical } \\
\text { parameter }\end{array}$ & $\begin{array}{l}\text { high pressure } \\
\text { water jet }\end{array}$ & $\begin{array}{c}\text { abrasive water } \\
\text { jet }\end{array}$ \\
\hline 1 & $\begin{array}{l}\text { operating } \\
\text { pressure }\end{array}$ & $\leq 140 \mathrm{MPa}$ & $\leq 30 \mathrm{MPa}$ \\
\hline 2 & operating flow & $\leq 6 \mathrm{~L} / \mathrm{min}$ & $\leq 6 \mathrm{~L} / \mathrm{min}$ \\
\hline 3 & $\begin{array}{l}\text { pressure } \\
\text { regulation }\end{array}$ & $\begin{array}{c}\text { reciprocating } \\
\text { pump / adjustable }\end{array}$ & $\begin{array}{l}\text { plunger pump } \\
\text { Un-adjustable }\end{array}$ \\
\hline 4 & hole diameter & $1 \sim 1.5 \mathrm{~mm}$ & $0.8 \mathrm{~mm}$ \\
\hline 5 & $\begin{array}{l}\text { Abrasive } \\
\text { particle }\end{array}$ & 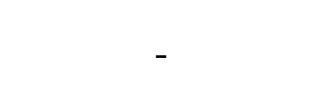 & $0.2 \mathrm{~mm}$ \\
\hline 6 & $\begin{array}{c}\text { Abrasive } \\
\text { concentration }\end{array}$ & - & $0 \sim 50 \%$ \\
\hline
\end{tabular}

\subsection{High-pressure water jet cutting technology}

As a new cutting technology, water jet cutting made use of ultrahigh-pressure water jet for power. While cutting shells body, it had advantage of high cutting efficiency and high precision. "Explosives Safety Manual" for US Army stated 
that when high-pressure water jets were used on the IHE, PBx-9404, LX-10 and other energetic materials, the jet pressure should be at a close range (less than $140 \mathrm{MPa}$ )[7], and not contain any abrasive. Through calculation, the theoretical velocity of jet is no more than $520 \mathrm{~m} / \mathrm{s}$, and the nozzle diameter is not more than $0.25 \mathrm{~mm}$, which has a certain reference value to selected water jet parameter for anti-riot ammunition. On the basis of it, HPA water jet cutting device manufactured by Shanghai Golden Arrow Company was chosen and implement to cut anti-riot ammunition as domestic successful precedents, shown in Figure 4. With the capable of ensuring security, the device used cutting system with CNC to basically meet the requirements of engineering destruction for explosive tear gas by strictly controlling the jet parameters, as shown Fig.3.
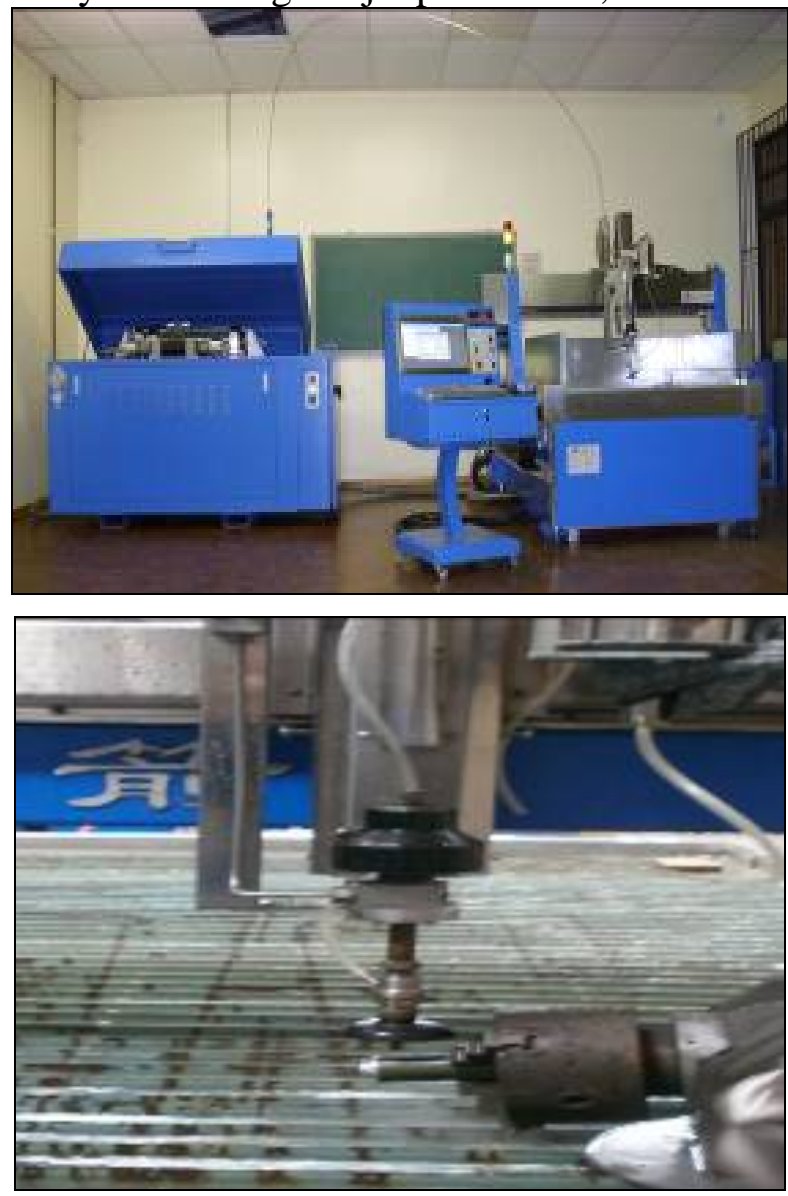

Fig.3: High press water jet cutting device

\section{Premixing abrasive water jet cutting technology}

Adding abrasive in water jet can significantly improve cutting the efficiency, and can reduce outlet pressure to about $30 \mathrm{MPa}$ under the similar result that can enable motorized the equipment. This low-pressure water cutting technology has a great application prospects in EOD and removal risk [8 10]. When facing on some ammunition that can not be removed or with more dangerous including the lifted insurance, the deformed shells and inherited bombs and etc, the advantages of its safety, efficiency and reliability can highlight.

Pre-mixed abrasive cutting technology can be adapted to a wide range. In general, the riot ammunition with metal projectile body should be priority given to the technology. The higher sense of ignition system, particularly, the ignition tube, primers, etc should avoid to be directly cut in order to prevent combustion or detonation. A portable water jet cutting systems produced by Baoding Atheros Company was chosen to carry out the cutting test for combustion tear gas. The cutting order should be noted that sensitive ignition component was first resection from the charge. Water jet should cut along with the middle section between the ignitron and ignition pills, shown in Figure 4, where the distance is about $5 \mathrm{~mm}$ and abrasive jet will not touch and pyrotechnics which 
can ensure the greatest degree of security. The second step was to recover the CS grain from projectile body, and then recycled or destroyed as the case.
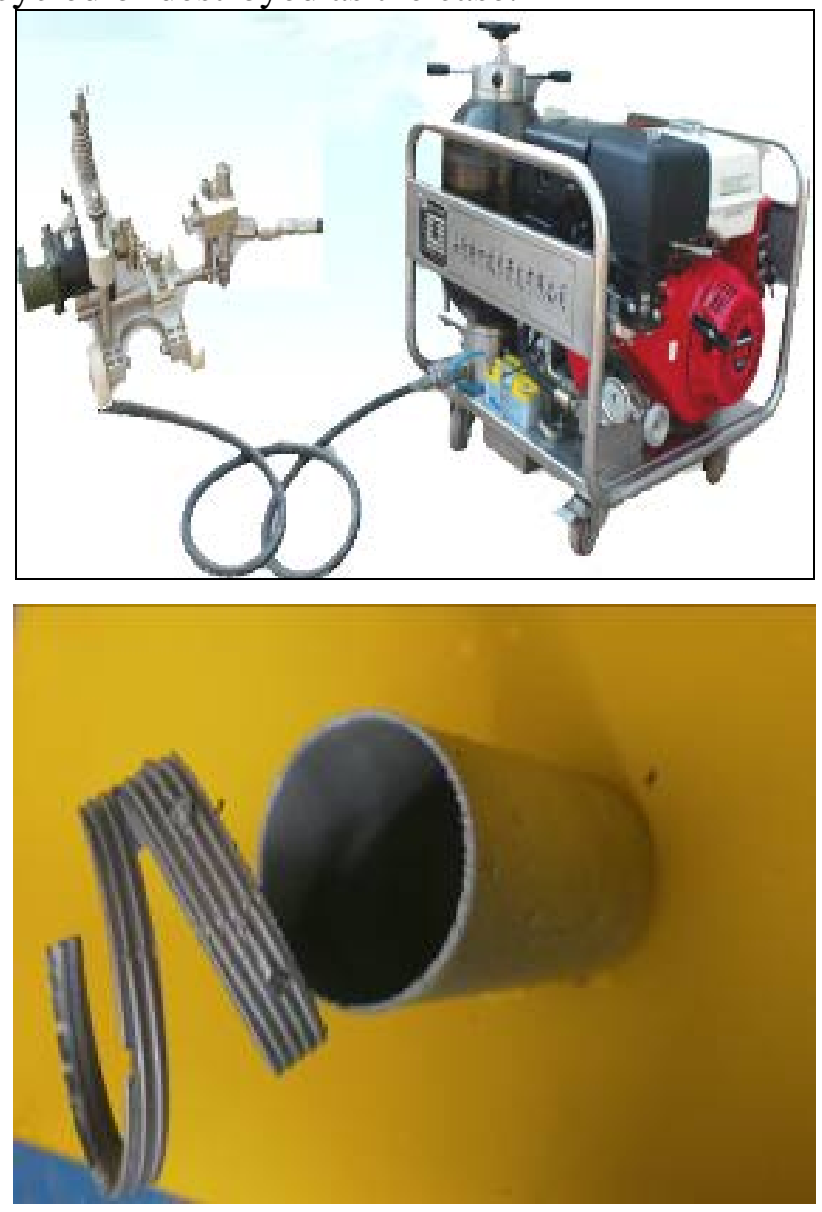

Fig.4: Abrasive water jet cutting device

\section{Conclusions}

In order to promote disposal work for obsolete anti-riot ammunition towards a trend with more secure, orderly, cost-effective and environmental friendly, methods and technology for the destruction were for a more in-depth study and exploration, and two key issues were drawn as the following conclusions:

(1) Disposal work for obsolete anti-riot ammunition must be based on the internal structure and mode of action of anti-riot ammunition, must ensure the process safety as a precondition.

(2) New methods of destruction of common ammunition innovative conventional methods to improve the efficiency of destruction. For example, using of water jet cutting technology fit for destruction of the abandoned anti-riot ammunition.

\section{Acknowledgement}

In this paper, the research was sponsored by the Nature Science Foundation of Shaanxi Province (Project No. 2014JM7284) and the Post-doctoral Science Foundation of China (Grant No. 2012M521945).

\section{Reference}

[1] Ma Yongzhong. Domestic Rs97-2 type combustion tear gas [J], light weapons, 2003, 7: 11-12.

[2] Yang Guang Ammunition of CAPF [M]. Xi'an: Engineering UniveRsity of CAPF, 2005. 
[3] Hubert Attaway, Old rich Mac hacek. Recycling of excess and demilitarized energetic materials in commercial explosive applications [R] .AD-A329686, 1997.

[4] Gong Yongxiao. Processing technology for abandoned general ammunition [M], Beijing: PLA Publishing House, 2004.

[5] Stephen W.Maloney Reuse of Ammonium Nitrate Wet Air Oxidation [R] US Army Corps EngineeRs CERL Technical Report, 1999, 09.

[6] Zhu Shuang. High pressure water jet cutting technology and application development [C], 50th anniversary Changsha Mining and Research Institute Proceedings, 2006, 10: 87-88.

[7] Xue Shengxiong. Pressure water jet technology engineering [M] Hefei: Hefei University of Technology, 2006.

[8] Gu Cheng Chu and He Yunhua. The analysis and research on cutting mechanism technology high-pressure water jet and cutting technology of abrasive water jet [J], mining machinery, 2004, 3: 48-52.

[9] Zhong Shuliang. Study on water jet cutting technology for explosives [D] Beijing: Chinese Academy of Engineering Physics, Doctoral Dissertation, 2006.

[10] Li Jinghai. Study on Explosives Pyrotechnics in processing and recycling of abandoned missile [J], defense technology base, 2007, 6 (6): 49-52. 